\title{
CLINICAL AND BIOLOGICAL EVOLUTION OF THE NEWBORN WITH THROMBOCYTOPENIA IN THE NEONATAL INTENSIVE CARE UNIT
}

\author{
Andrei Munteanu ${ }^{1,2}$, Aniko Manea ${ }^{1,2}$, Cristian Jinca ${ }^{2,3}$, Marioara Boia ${ }^{1,2}$
}

\section{Abstract}

Introduction: Thrombocytopenia is one of the most common haematological disorders detected in the newborn period, especially in neonates admitted to intensive care units and usually indicates an underlying pathological process. Objectives: To determine the etiology, the time of onset, the clinical characteristics and the evolution of newborns with thrombocytopenia. Material and method: 97 newborns with platelet counts $(<150,000 / \mu \mathrm{l})$ were selected from those admitted to the Neonatology section of the Timisoara Children's Emergency Hospital "Louis Turcanu" for a period of 3 years. The determination of the initial values of platelets was performed at the hospitalization and then daily monitoring or even twice daily in the case of newborns with severe thrombocytopenia. Results: Grade IV thrombocytopenia $(<25000 / \mu \mathrm{l})$ was present in $13.4 \%$ of cases, grade III $(25000-50,000 / \mu \mathrm{L})$ in $16.5 \%$ of cases, grade II $(50,000-75,000 / \mu \mathrm{L})$ in $9,3 \%$ of cases and grade I $(75,000-150000 / \mu \mathrm{L})$ in $60.8 \%$ of cases. Most of the newborns $(55.7 \%)$ were premature and the major causes of thrombocytopenia were sepsis, in $69.1 \%$ of cases and hypoxic suffering in $66 \%$ of cases. Mortality was $12.6 \%$ (n $=$ 14). Conclusion: Thrombocytopenia occurring in newborns admitted to the neonatal intensive care unit is not a negative prognostic factor but rather a marker of severity of the underlying pathology.

Abbreviations: HIE, hypoxic ischemic encephalopathy; SGA, small for gestational age; AGA, appropriate for gestational age;

Keywords: thrombocytopenia, newborns, infections, hypoxia

\section{Introduction}

Studies of fetal blood obtained by cordocentesis show that the mean fetal platelet count reaches $150 \times 10^{9} / \mu \mathrm{L}$ by the end of the first trimester of pregnancy and increases above $150 \times 10^{9} / \mu \mathrm{L}$ by end of the second trimester. Several population studies also show that $>98 \%$ of term neonates born to mothers with normal platelet counts have platelets above $150 \times 10^{9} / \mu \mathrm{L}$ at birth. Therefore thrombocytopenia in a neonate of any viable gestational age can be defined as a platelet count of $<150 \times 10^{9} / \mu \mathrm{L}$ The incidence is $1-5 \%$ of newborns and $22-35 \%$ of newborns admitted to neonatal intensive care units[1,2,3,4].

The risk of severe thrombocytopenia is higher in preterm infants. In newborns with extremely low birth weight ( $<1,000$ grams), the incidence of thrombocytopenia is greater than $70 \%$ and the incidence of severe thrombocytopenia $(<50,000 / \mu \mathrm{L})$ is $40 \%$ [5]. The period of onset of thrombocytopenia can be: early (within 72 hours after birth) and late (after 72 hours of life).

Causes of early thrombocytopenia are placental insufficiency, perinatal asphyxia, autoimmune or alloimmune. Hypoxia-ischemia (HI) is a contributing factor to neonatal morbidity and mortality, often leading to chronic neurological disorders and disabilities, such as mental retardation, motor and behavioral developmental issues, cerebral palsy, seizure, and epilepsy. Newborns with mild HIE (grade I) have a favorable evolution. Approximately $80 \%$ of the patients with grade II encephalopathy recover; however, the mortality rate is 3 and $20-45 \%$ have neurological sequelae. Patients with severe HIE (grade III) have a mortality rate of $50 \%$ and survivors present severe neurological consequences [6]. The incidence of HIE ranges from 1 to 8 per 1,000 live births in developed countries and is as high as 26 per 1,000 live births in underdeveloped countries [7].

Manifestations of HIE involve heart rhythm disorders, basic acid balance disorders $(\mathrm{pH}<7.0$ or basic deficiency $\geq 12 \mathrm{mmol} / \mathrm{l}$ ), low Apgar index, amniotic fluid impregnated with meconium or the need for respiratory support in the first few minutes of postnatal life [8].

${ }^{1}$ Department XII Obstetrics - Gynecology, Discipline of Neonatology and Childcare, "Victor Babes" University of Medicine and Pharmacy Timișoara, Romania, Eftimie Murgu Sq. no.2, 300041, Timişoara, RO

2"Louis Turcanu" Children's Emergency Hospital, Str. Iosif Nemoianu no.2, 300011, Timisoara, Romania

${ }^{3}$ Department XI Pediatrics, Discipline III Pediatrics, "Victor Babes" University of Medicine and Pharmacy Timișoara, Romania, Eftimie Murgu Sq. no.2, 300041, Timisoara, RO

E-mail: andrei.munteanu30@yahoo.com, aniko180798@yahoo.com, cristian_jinca@yahoo.com, marianaboia@yahoo.com 
The redistribution of cerebral blood flow induced by asphyxia is the main post asphyxiation change. Brain injury results from hypoxia and ischemia. As a result of asphyxia, cardiac output is compensated by redistribution, thus increasing cerebral blood flow. If hypoxia persists, this self regulatory mechanism is no longer effective, resulting in decreased heart rate, with systemic hypotension and decreased cerebral flow leading to brain damage. At the cellular level, oxygen depletion blocks oxidative phosphorylation resulting in an anaerobic metabolism, which is energy inefficient, resulting in: i) Rapid depletion of phosphate reserves, including adenosine triphosphate, ii) accumulation of lactic acid and iii) inability to maintain cellular functions.

Severe maternal autoimmune thrombocytopenic disease, before or during pregnancy, was associated with an increased risk of severe fetal thrombocytopenia [9].

Late onset of thrombocytopenia is most commonly caused by infections / septicemia and necrotizing enterocolitis. Sometimes the causes of thrombocytopenia are prenatal viral infections (Cytomegalovirus, Toxoplasmosis, Rubella, HIV), perinatal bacterial infections (Group B streptococcus, Escherichia coli and Haemophilus Influenzae), or aneuploidy (especially trisomy 18, 13 and 21) $[1,10]$.

Thrombocytopenia is usually seen with Gram positive septicemia as compared to Gram negative septicemia and low platelet is usually seen even before the pathogens are cultured from the blood. Therefore, thrombocytopenia may be considered as an important and early tool in diagnosis of septicemia in neonates [11].

Also among the factors that cause thrombocytopenia were incriminated in the literature also $\mathrm{H} 2$ antagonists - a case of severe thrombocytopenia induced by Ranitidine [4] and also by Vancomycin [12] has been described.

Previous detailed studies have attempted to define the mechanisms by which these conditions cause thrombocytopenia, but, until recently, the mechanism underlying many neonatal thrombocytopenias remained unknown [13].

The rather complex process of platelet production and release can be schematically represented as being made up of four main stages:

1) the production of thrombopoietic cytokines, mostly thrombopoietin, which is produced in the liver and is the main regulator of platelet production in humans [14]. Platelets are involved in hemostasis, influence the coagulation cascade and are the main source of many biologically active substances [15].

2) proliferation of megakaryocyte progenitors;

3) maturation of megakaryocytes in large polypoid cells capable of producing platelets;

4) release of platelets into circulation. Megakaryocytes in newborns are smaller and less mature than adult megakaryocytes, and smaller megakaryocytes are known to produce fewer platelets than larger and more mature megakaryocytes. This developmental feature may limit the ability of newborns to increase platelet production in response to platelet consumption. In addition, preterm infants appear to have relatively low levels of thrombopoietin during thrombocytopenia, which may limit their ability to rapidly regulate platelet production during increased platelet consumption [16]. Thrombopoietin is elevated during hypoxia. The number of platelets is negatively correlated with thrombopoietin levels on days 1 , 3 and 7 of life in hypoxic infants [17].

Current evidence suggests that most platelet destruction in the newborn is immunologically mediated. It is shown that $15 \%$ to $20 \%$ of all neonatal thrombocytopenia present at birth results from transplacental passage of allo and / or autoantibodies [10]. Alloimmunization is the most common cause of severe thrombocytopenia [18].

Prematures babys born from the mother with preeclampsia who develops early bacterial sepsis and the baby with intrauterine growth restriction who develops necrotizing enterocolitis can both become thrombocytopenic (after pre-eclampsia or intrauterine growth restriction), combined with thrombocyte intake (during sepsis or necrotizing enterocolitis) [13].

It was found a strong correlation between delivery by cesarean section and thrombocytopenia. Disseminated intravascular coagulation occurs during sepsis and is found in $10 \%-15 \%$ of thrombocytopenia cases admitted in neonatal intensive care [19].

In some studies it was concluded that birth weight and head circumference in infants with thrombocytopenia were significantly lower than in infants without thrombocytopenia. There was also a smaller number of hematopoietic progenitor cells in the blood from the umbilical cord of the SGA compared to the AGA [20].

\section{Material and method:}

This is a retrospective, cross-sectional cohort study carried out in the Neonatology Section of the Emergency Hospital for Children "Louis Turcanu" Timisoara. The study was conducted for a period of 3 years, from 01.01.2016 to 31.12.2018. The study included 97 newborns with a birth weight between 450 grams and 4900 grams.

Inclusion criteria:

1) Newborn suffering from birth asphyxia and subsequently developing hypoxic ischaemic encephalopathy or newborns with neonatal infection.

2) Newborn who fulfill the case definition criteria

3) Birth weight 450 grams and 4900 grams

4) Neonates surviving more than $24 \mathrm{hrs}$.

Exclusion Criteria:

1) incomplete data about the patient, information unavailable in the computer system of the hospital unit

2) major congenital anomalies, birth trauma

3) newborn borns to mothers having major diseases like malaria, severe anaemia, pre eclamsia/eclamsia, thyroid disorder, idiopathic purpura, placental disorder like vascular thrombosis, abruptio placenta

4) H/o maternal intake of any drugs causing bone marrow suppression/ thrombocytopenia

5) Newborn with congenital leukaemia, those having exchange transfusion. 


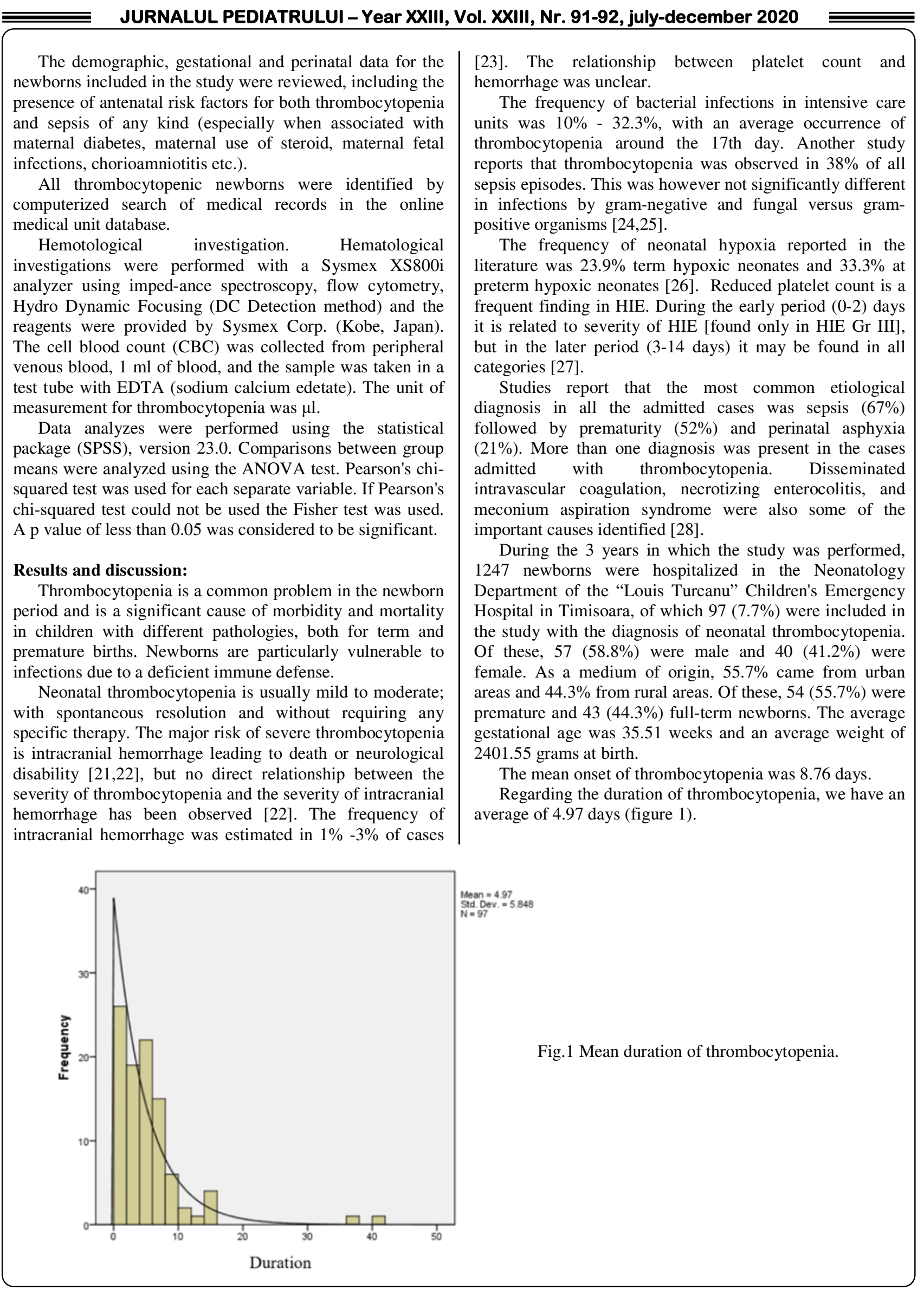


predominated both in the first 72 hours and at more than 72 hours (table 1).

The mean value of platelets was $93.510 / \mu 1$. Depending on the value of platelets, patients were classified in severity, so 59 newborns $(60.8 \%)$ were classified in the first grade of severity $(75000-150000 / \mu \mathrm{L}), 9$ newborns $(9.3 \%)$ in grade II $(50000-75000 / \mu \mathrm{L}), 16$ newborns $(16.5 \%)$ in grade III $(25000-50000 / \mu \mathrm{L})$ and 13 newborns $(13.4 \%)$ in grade IV $(<25000 / \mu \mathrm{L})$ (figure 2$)$.

Table 1. Duration of thrombocytopenia.

\begin{tabular}{|l|l|l|l|l|}
\hline $\begin{array}{l}\text { Study on neonatal } \\
\text { thrombocytopenia in } \\
\text { NICUs }\end{array}$ & $<\mathbf{7 2}$ hours & & $>\mathbf{7 2}$ hours & \\
\hline & Moderate & Severe & Moderate & Severe \\
\hline Castle et al $^{(24)}$ & $66.11 \%$ & $47.01 \%$ & $39.24 \%$ & $52.23 \%$ \\
\hline${\text { Rajeev } \text { mehta }^{(24)}}_{\text {Patil et al }}^{(24)}$ & $13 \%$ & $20 \%$ & $36.2 \%$ & $51 \%$ \\
\hline Our study & $73.11 \%$ & $44.68 \%$ & $26.88 \%$ & $55.31 \%$ \\
\hline
\end{tabular}

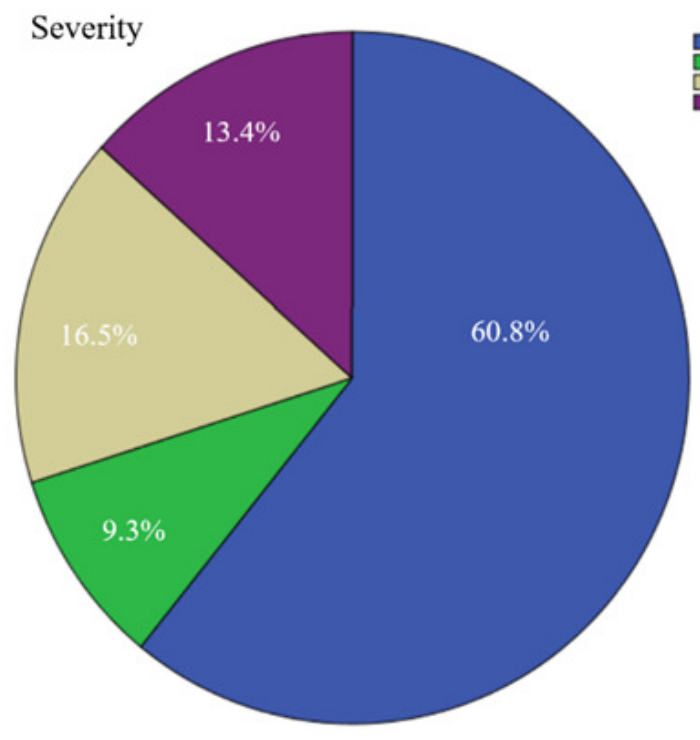

Fig.2 Severity of thrombocytopenia.

Knowing that thrombocytopenia occurs more frequently in the first 3 days in patients with hypoxia and after 3 days in patients with infections or ulceronecrotic enterocolitis, it was observed that the onset of thrombocytopenia in the first 3 days of life was found in 62 newborns $(63.9 \%)$, and after 3 days of life in 35 newborns (36.1\%).
Hypoxia was found in $66 \%$ of thrombocytopenic patients, of whom $51.5 \%$ were premature newborns, predominantly male $(57.5 \%)$ (Table 2$)$.

Infections were detected in $69.1 \%$ of newborns, and hemorrhagic pathology or hemorrhagic syndromes were observed in $37.1 \%$ of newborns (table 3 ).

Tabel 2. Prevalence of hypoxemia.

\begin{tabular}{|l|l|}
\hline Studies on neonatal thrombocytopenia in NICUs & Prevalence of hypoxemia \\
\hline Nadkarniet $\mathrm{al}^{(21)}$ & $23.9 \%$ term hypoxic neonates \\
& $33.3 \%$ preterm hypoxic neonates \\
\hline Our study & $\mathbf{4 8 . 5 \%}$ term hypoxic neonates \\
& $\mathbf{5 1 . 5 \%}$ preterm hypoxic neonates \\
\hline
\end{tabular}




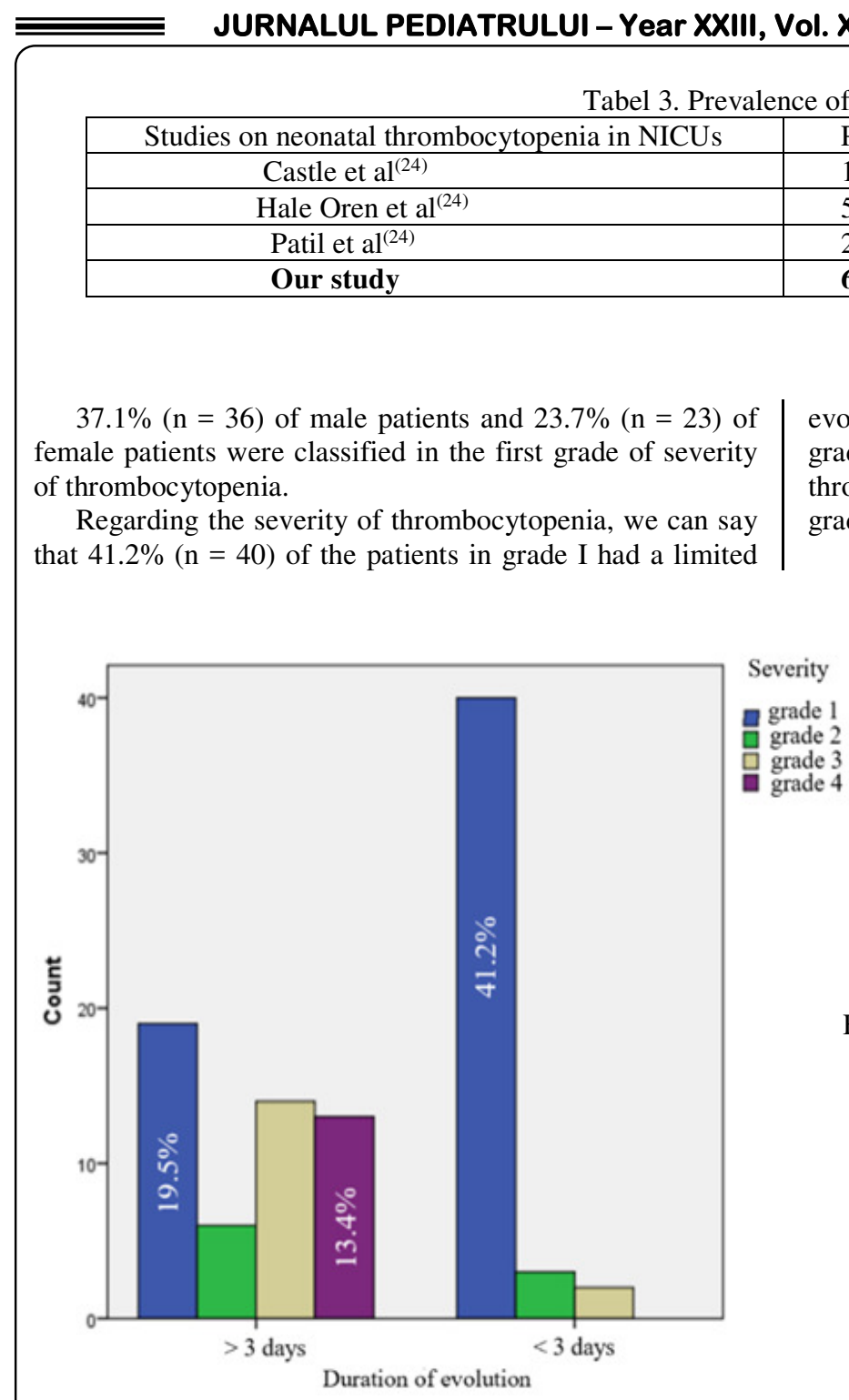

Knowing that $53.6 \%(\mathrm{n}=52)$ of patients with thrombocytopenia had a duration of evolution over 3 days, $30.9 \%(n=30)$ were male and $22.6 \%(n=22)$ female. In both the group with evolution less than 3 days and in the group with evolution over 3 days, male patients were predominant.

Prematurity was found in $55.7 \%(\mathrm{n}=54)$ of cases, predominating male $-51.8 \%$ compared to $48.1 \%$ females. We observe that in case of the study group with evolution over 3 days of thrombocytopenia, 34\% $(n=33)$ were born prematurely, while in the case of the group with evolution under 3 days, $24.7 \%(n=24)$ were newborn at term. Knowing that in the first 3 days thrombocytopenia is more common due to hypoxia, we can say that in newborns at term thrombocytopenia the date of hypoxia was more common in the first 3 days (figure 4). Regarding the severity of thrombocytopenia, the vast majority of premature infants evolution in less than 3 days, and $19.5 \%(n=19)$ of those in grade I had an evolution more than 3 days of thrombocytopenia, while the evolution of all patients in grade IV severity (13.4\%) was over 3 days (figure 3 ).
Fig.3 Severity depending on the duration of evolution.

$48.4 \%(n=31)$ were classified in the first grade of severity (figure 5).

Hypoxia was found in $66 \%(\mathrm{n}=64)$ of thrombocytopenic patients, $54.6 \%(\mathrm{n}=35)$ were male and $45.4 \%(n=29)$ female. Analyzing the incidence of hypoxia depending on the duration of thrombocytopenia, we observe that in patients with less than 3 days of evolution, $45.3 \%$ (n = 29) were hypoxic, while in the case of the group with more than 3 days of thrombocytopenia, $54.6 \%(n=35)$ were hypoxic (figure 6). The vast majority of $40.2 \%$ hypoxic patients $(n=39)$ were classified in the first grade of severity (figure 7).

In figure 8 are supported the previously reported, that $68.7 \%(n=44)$ of premature babies were hypoxic.

As values with statistical significance $(p<0.05)$, it was observed that in the case of female newborns, thrombocytopenia started on average at 4.43 days; while in male newborns at 11.8 days (table 4 ). 


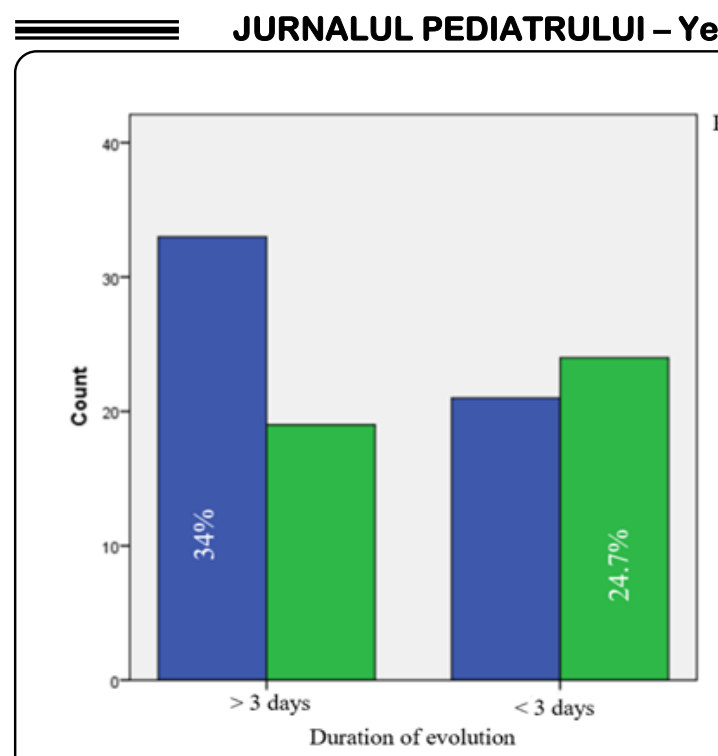

Fig.4 Duration of evolution in premature newborns.

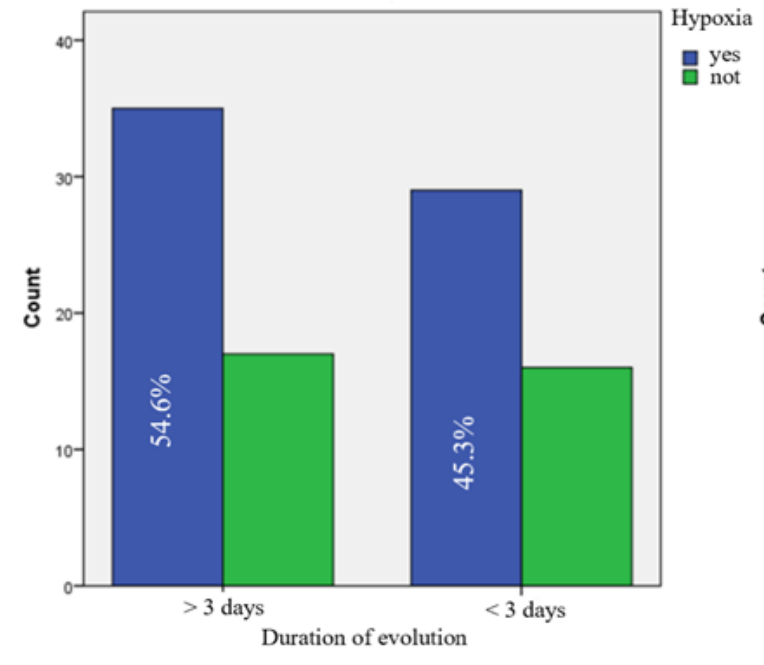

Fig.6 Duration of thrombocytopenia in hypoxic newborns.

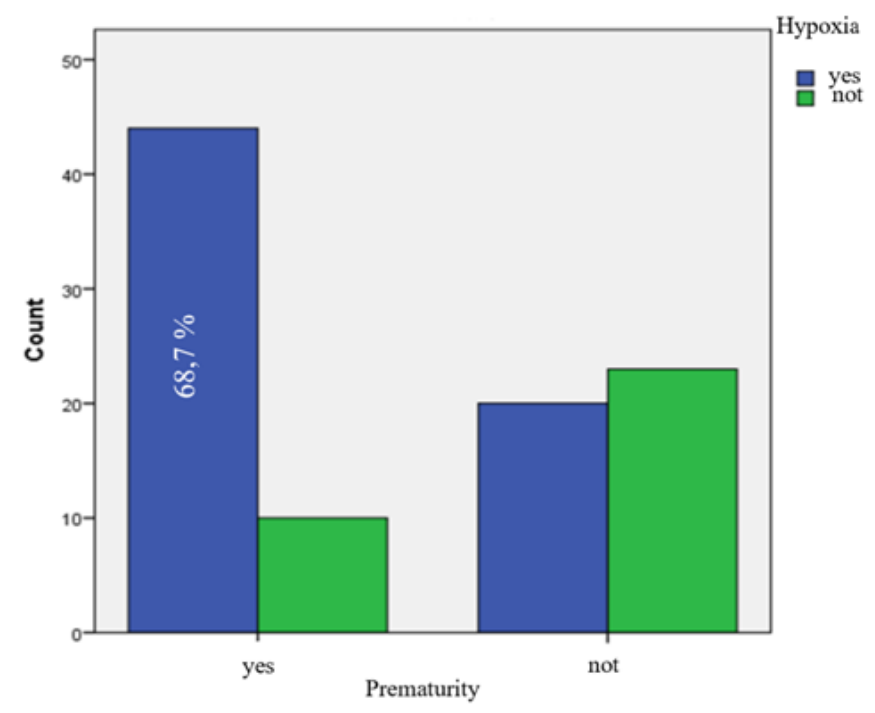

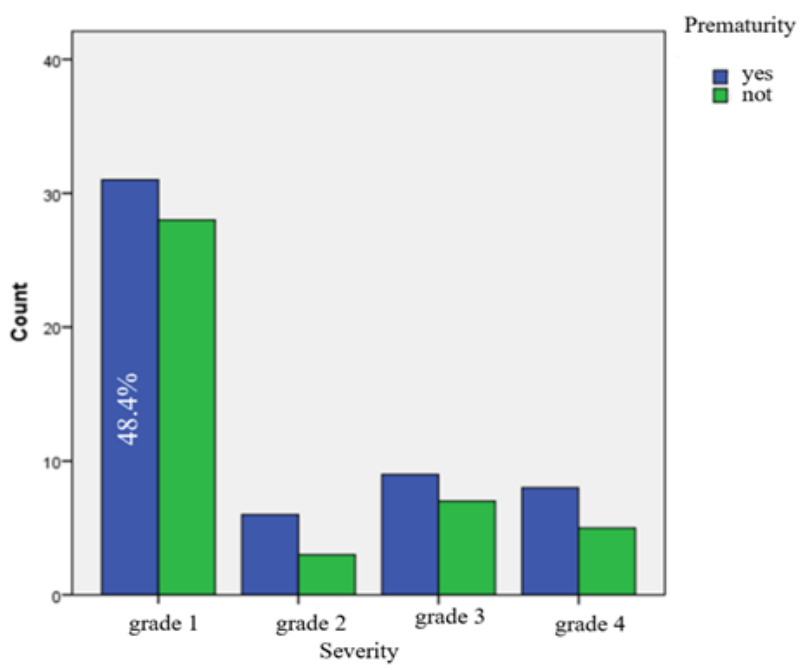

Fig.5 Severity of thrombocytopenia in premature infants.

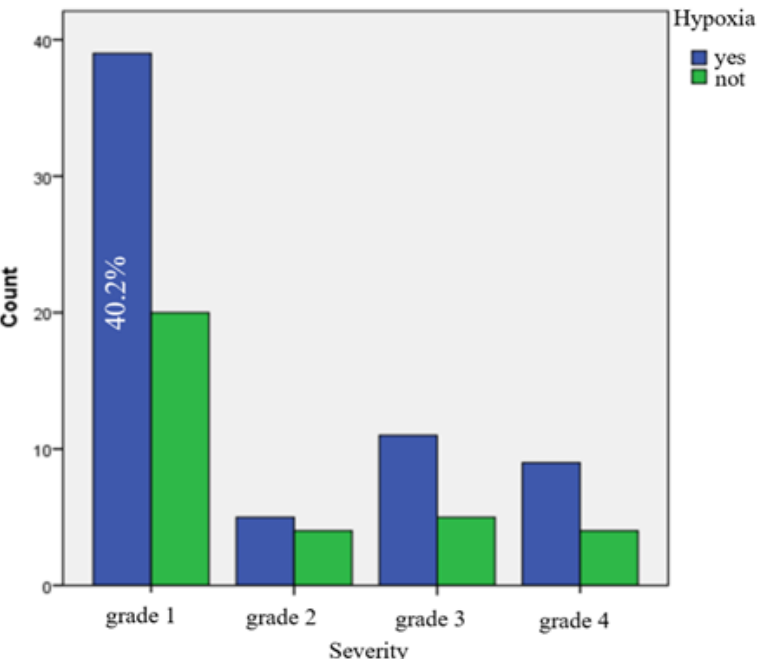

Fig.7 Severity of thrombocytopenia in hypoxic newborns.

Fig.8 Prematurity in hypoxic newborns. 


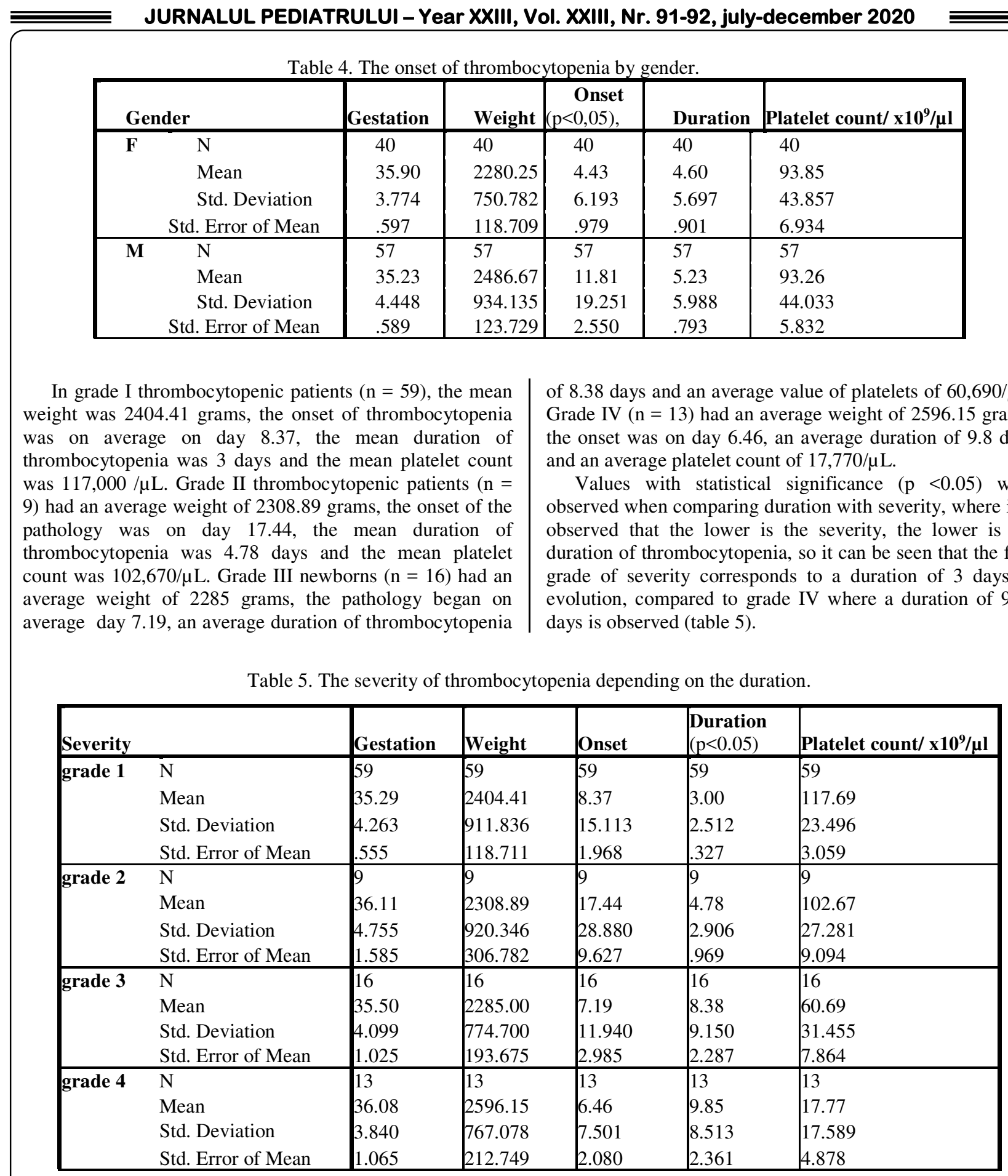

Depending on the duration of thrombocytopenia, patients were divided into two groups; lasting less than 3 days and lasting more than 3 days.

Thus with the statistically significant value $(\mathrm{p}<0.05)$ it was observed that in the case of patients in whom thrombocytopenia lasted up to 3 days $(n=45)$ the average onset was on day 12.36; the mean duration of thrombocytopenia was 1.6 days and the average platelet count was $115,640 / \mu \mathrm{L}$. In neonates in whom thrombocytopenia lasted more than 3 days $(n=52)$, it was observed that on average the onset was in day 5.65, the mean duration of thrombocytopenia was 7.88 days and the mean value of platelet count was $74,350 / \mu \mathrm{L}$ (table 6). 


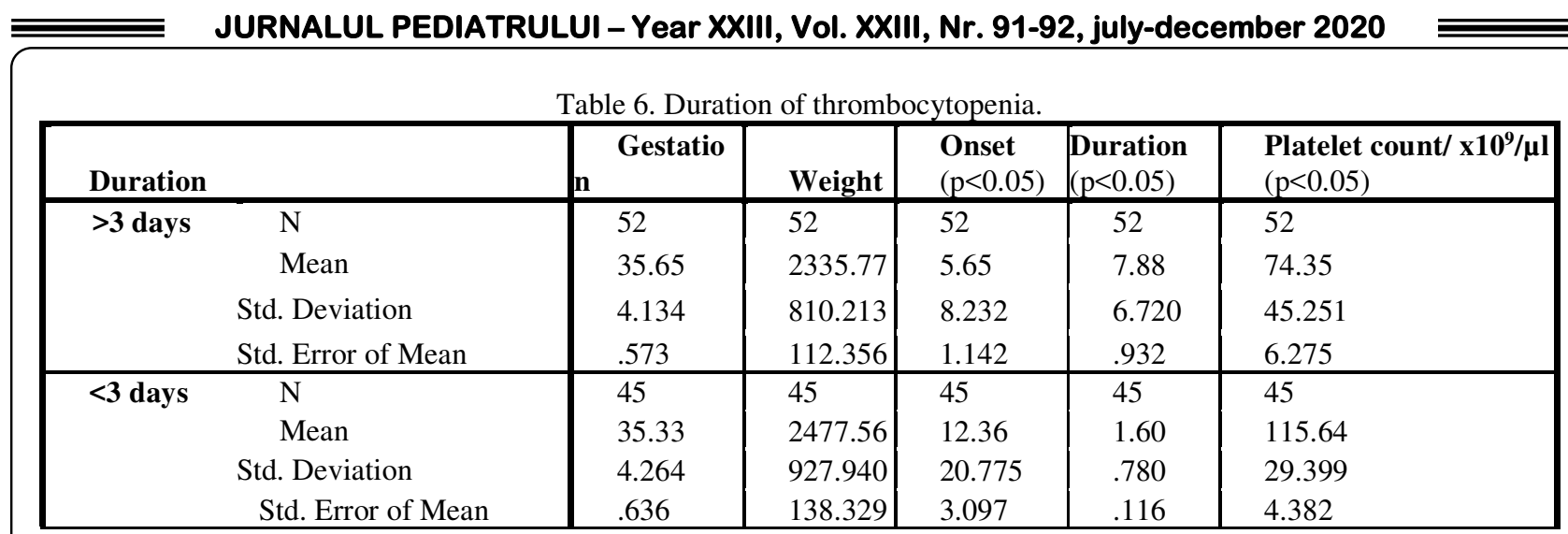

In the case of hypoxic patients $(n=64)$ compared to those who did not suffer from hypoxia, with statistically significant value $(\mathrm{p}<0.05)$, it was observed that the average birth weight was 2197.66 grams, the average gestational age was 34.42 weeks and onset of thrombocytopenia on day
5.33. In the case of newborns without hypoxic distress, it was observed that the average birth weight was 2796.97 grams, the average gestational age was 37.6 weeks and the onset of thrombocytopenia on day 15.42 (Table 7).

Table 7. Evaluation of hypoxic newborns.

\begin{tabular}{|c|c|c|c|c|c|c|}
\hline \multicolumn{2}{|c|}{ Hypoxia } & $\begin{array}{l}\text { Gestation } \\
(\mathrm{p}<0.05)\end{array}$ & $\begin{array}{l}\text { Weight } \\
(\mathrm{p}<0.05)\end{array}$ & $\begin{array}{r}\text { Onset } \\
(p<0.05)\end{array}$ & Duration & Platelet count $/ \times 10^{9} / \mu \mathrm{l}$ \\
\hline \multirow[t]{4}{*}{ yes } & $\mathrm{N}$ & 64 & 64 & 64 & 64 & 64 \\
\hline & Mean & 34.42 & 2197.66 & 5.33 & 5.09 & 91.89 \\
\hline & Std. Deviation & 4.305 & 897.327 & 12.947 & 5.628 & 44.687 \\
\hline & Std. Error of Mean & .538 & 112.166 & 1.618 & .703 & 5.586 \\
\hline \multirow[t]{4}{*}{ not } & $\mathrm{N}$ & 33 & 33 & 33 & 33 & 33 \\
\hline & Mean & 37.61 & 2796.97 & 15.42 & 4.73 & 96.64 \\
\hline & Std. Deviation & 2.989 & 644.765 & 18.329 & 6.336 & 42.319 \\
\hline & Std. Error of Mean & .520 & 112.239 & 3.191 & 1.103 & 7.367 \\
\hline
\end{tabular}

It was also observed that in the case of hypoxic patients the mean duration of thrombocytopenia was 5.09 days with a mean platelet count of $91,890 / \mu \mathrm{L}$, and in neonates without hypoxic distress, the mean duration of thrombocytopenia was 4.7 days with a mean platelet count of $96,640 / \mu \mathrm{L}$.
In patients with infection $(n=67)$, the mean time to onset of thrombocytopenia was 10.99 days, while in patients without infection $(\mathrm{n}=30)$ the mean time to onset of thrombocytopenia was 3.80 days, with a mean duration smilara and an average platelet value approximately equal (Table 8).

Table 8. Evaluation of thrombocytopenic infections in newborns.

\begin{tabular}{|c|c|c|c|c|c|c|}
\hline \multicolumn{2}{|c|}{ Infections } & Gestation & Weight & Onset & Duration & Platelet count $/ \times 10^{9} / \mu \mathrm{l}$ \\
\hline \multirow[t]{4}{*}{ yes } & $\mathrm{N}$ & 67 & 67 & 67 & 67 & 67 \\
\hline & Mean & 35.55 & 2433.13 & 10.99 & 4.75 & 90.85 \\
\hline & Std. Deviation & 4.415 & 914.965 & 17.990 & 5.417 & 44.618 \\
\hline & Std. Error of Mean & .539 & 111.781 & 2.198 & .662 & 5.451 \\
\hline \multirow[t]{4}{*}{ not } & $\mathrm{N}$ & 30 & 30 & 30 & 30 & 30 \\
\hline & Mean & 35.40 & 2331.00 & 3.80 & 5.47 & 99.43 \\
\hline & Std. Deviation & 3.654 & 751.598 & 6.155 & 6.786 & 41.807 \\
\hline & Std. Error of Mean & .667 & 137.222 & 1.124 & 1.239 & 7.633 \\
\hline
\end{tabular}




\section{Conclusion}

The most common cause of early-onset thrombocytopenia is fetal hypoxia; most often it is selflimited and rarely severe.

After 72 hours, the most common cause of thrombocytopenia among newborns admitted to the intensive care unit is infectious pathology.

In our study, the hypoxic infants with whom thrombocytopenia was associated were predominant in males.

In the case of hypoxic patients, thrombocytopenia started earlier, had a longer duration and a higher grade of severity compared to newborns without hypoxic suffering.

It was observed that the more severe are the form of thrombocytopenia, the longer is the regeneration period. As the severity of thrombocytopenia was lower, the duration of thrombocytopenia was shorter and appeared later. The onset of thrombocytopenia among the patients in study was 8.7 days and with an average duration of 4.97 days.

Thrombocytopenia occurring in neonates admitted to the neonatal intensive care unit is not a negative prognostic factor but rather a marker of severity of the underlying pathology.

\section{Acknowledgements}

Professional editing, linguistic and technical assistance was performed by Irina Radu.

\section{Funding}

No funding was received.

\section{Availability of data and materials}

The datasets used and/or analyzed during the current study are available from the corresponding author on reasonable request.

\section{Authors' contributions}

AIM and MB conceived and designed the study; AIM and AMM collected the data. AMM and CMJ analyzed the data; AIM and CMJ edited the figures and AIM, AMM and CMJ drafted the manuscript. $\mathrm{MB}$ revised the manuscript critically for important intellectual content. All authors contributed to the data interpretation and approved the submitted version.

\section{Ethics approval and consent to participate}

Approval of the local ethics committee (Ethics Committee for Scientific Research of the Emergency Hospital for Children 'Louis Turcanu) was obtained prior to starting the study. Parental or caregiver consent was obtained where applicable. This publication and the database does not contain personal data, does not compromise anonymity or confidentiality or breach local data protection laws.

\section{Patient consent for publication}

Not applicable.

\section{Competing interests}

The authors declare that they have no competing interests.

\section{References}

1. Roberts I, Stanworth S, Murray NA. Thrombocytopenia in the Neonate. Blood Rev. 2008;22(4):173-86.

2. Jerónimo M, Azenha C, Mesquita J, Pereira DF. A rare manifestation of neonatal alloimmune thrombocytopaenia. BMJ Case Rep. 2014;10-3.

3. von Lindern JS, van den Bruele T, Lopriore E, Walther FJ. Thrombocytopenia in neonates and the risk of intraventricular hemorrhage: A retrospective cohort study. BMC Pediatr [Internet]. 2011;11(1):16. Available from: http://www.biomedcentral.com/1471$2431 / 11 / 16$

4. Hoste L, George I. Ranitidine-induced Thrombocytopenia in a Neonate - A Case Report and Review of Literature. J Pediatr Pharmacol Ther. 2019;24(1):66-71.

5. Yurdakök M. Immune thrombocytopenia in the newborn. J Pediatr Neonatal Individ Med. 2017;6(1):110.

6. Chaparro-Huerta V, Flores-Soto ME, Merin Sigala ME, Barrera de León JC, Lemus-Varela M de L, TorresMendoza BM de G, et al. Proinflammatory Cytokines, Enolase and S-100 as Early Biochemical Indicators of Hypoxic-Ischemic Encephalopathy Following Perinatal
Asphyxia in Newborns. Pediatr Neonatol. 2017;58(1):70-6.

7. Douglas-Escobar M, Weiss MD. Hypoxic-Ischemic Encephalopathy A Review for the Clinician. JAMA Pediatr. 2015;169(4):397-403.

8. Allen KA, Brandon DH. Hypoxic Ischemic Encephalopathy: Pathophysiology and Experimental Treatments. Newborn Infant Nurs Rev [Internet]. 2011;11(3):125-33. Available from: http://dx.doi.org/10.1053/j.nainr.2011.07.004

9. Valat AS, Caulier MT, Devos P, Rugeri L, Wibaut B, Vaast $\mathrm{P}$, et al. Relationships between severe neonatal thrombocytopenia and maternal characteristics in pregnancies associated with autoimmune thrombocytopenia. Br J Haematol. 1998;103(2):397401.

10. Roberts IAG, Murray NA. Neonatal thrombocytopenia: New insights into pathogenesis and implications for clinical management. Curr Opin Pediatr. 2001;13(1):16-21.

11. Arif SH, Ahmad I, Ali SM, Khan HM. Thrombocytopenia and bacterial sepsis in neonates. Indian J Hematol Blood Transfus. 2012;28(3):147-51. 


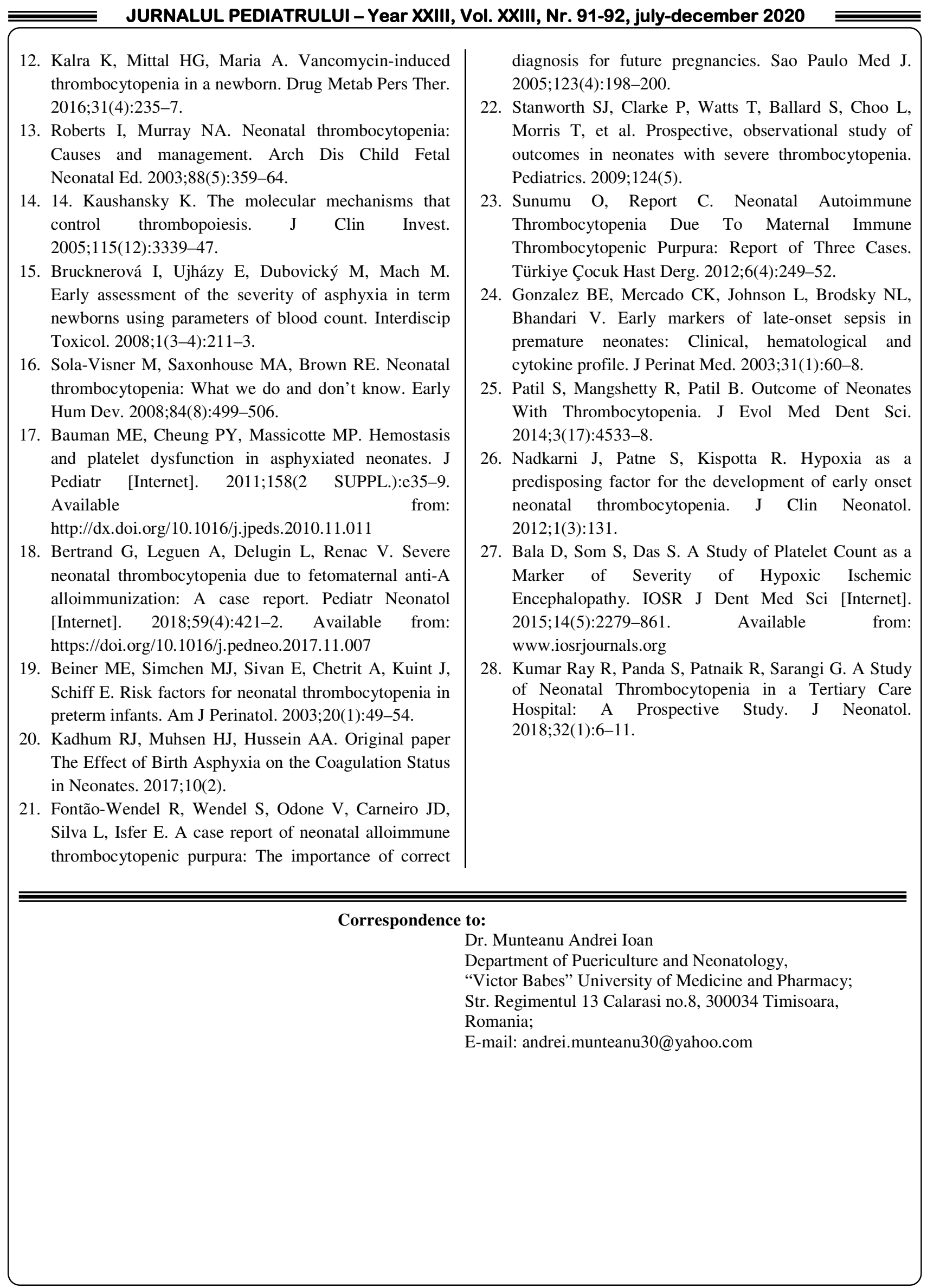

